\title{
INTERVIEW WITH JUDE F. CLANCY, MD
}

\section{ANGELINA WAGNER}

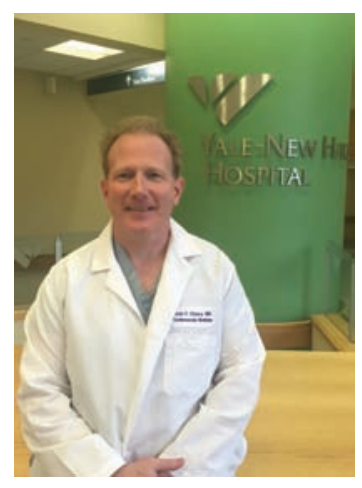

\section{Introduction}

Lead extraction procedures occur because of the need to remove one or more leads from inside the heart. Infections at the implantable cardiac device site, blockage of the vein, excess formation of scar tissue, or damage to the inside or outside of the lead are determinants for these procedures. Although lead extractions are not frequently performed, awareness of complications is important. This includes superior vena cava (SVC) tears. A tear in the SVC during lead extraction is a rare event, but can result in massive hemorrhage and hemodynamic instability. New technology and techniques give investigators control, safety and stability during lead extraction procedures.

Dr. Jude F. Clancy is an electrophysiologist in New Haven, Connecticut and Assistant Professor, Department of Internal Medicine at Yale University School of Medicine. Clancy received his medical degree from University of Massachusetts Medical School and has been in practice for more than 10 years.

We speak with Dr. Jude F. Clancy, MD about his perspectives and discernments on how new technology and techniques are being proposed with the use of lead extraction.

Wagner: Tears in the SVC during lead extraction are rare events but what emerging techniques and technologies are being conducted within this area?

Clancy: When looking at a problem like SVC tears, even though it doesn't happen very often, when it does happen, it's a potentially major devastating event. We can approach the problem from two sides. We can make the best equipment possible so as to minimize the possibility of a tear. And then even though the best equipment is possible, there's nothing at zero percent risk. However, once there's a tear, what can be done in terms of tears and resuscitation to limit mortality and reduce mortality hopefully to zero? A new rescue device, the Bridge ${ }^{\mathrm{TM}}$ Occlusion Balloon (Spectranetics, Colorado Springs, CO), fits into that second part, where unfortunately there's already a tear which usually results in massive bleeding and the patients approximately $50 \%$ of the time do not recover from this. The Bridge ${ }^{\mathrm{TM}}$ Occlusion Balloon (Spectranetics, Colorado Springs, CO) a FDA approved compliant balloon can be deployed in the SVC to basically tamponade the tear and stabilize the patient until a definitive operation can be done within a couple minutes. We then definitively fix the tear and help the patient.

\section{Wagner: What has been your personal experience with new devices especially in the operating room?}

Clancy: I've done a lot of studies in the porcine model. The Bridge ${ }^{\mathrm{TM}}$ Occlusion Balloon (Spectranetics, Colorado Springs, CO) was developed in that model and it was extremely impressive how it functioned there. These large tears would be made and the balloon would deploy in a pool of blood and then only have a trickle of blood coming out which was very impressive to see. It's very reassuring having this rescue device to impact this area and prevent massive blood loss. Translating to the human side, physicians have put the balloon up and inflated the balloon to show that it can occlude, which is brilliant and that's what we need it to do. Since it was recently FDA approved, it's just rolling out and so there hasn't been use of it in an event yet. These types of developments in technology can't be really tested in the same manner as say a catheter because it's a rescue device. I wish it would never happen but we have to wait until a bad event to see how the balloon functions in that scenario. 
Wagner: What have been the main issues and controversies in lead extraction within the industry?

Clancy: I think that even though the mortality associated with the procedure is extremely low at about $0.2 \%$, there's a preconceived notion that lead extraction is a very high risk procedure. When we look at the risk of lead extractions compared to other procedures that we do every day, such as one of the most common procedures we do is atrial fibrillation (AF) ablation, the risk and adverse outcomes are higher and mortality is slightly higher, too. So it's not fivefold different than any other procedure. We are trying to dispel those myths, both on the patient side and the referral side, as well as the operator side because we want the patients to get the appropriate care. We know that the patients who don't get lead extractions that need extractions suffer and we don't want fear of the procedure preventing them from getting the appropriate care. I think the Bridge ${ }^{\mathrm{TM}}$ Occlusion Balloon (Spectranetics, Colorado Springs, CO) is one more tool in addition to the laser and the TightRail mechanical device (Spectranetics, Colorado Springs, CO). As we continue to see new equipment coming down the pipe, it will make lead extractions safer, and the safer we can make this procedure, the more patients will be benefited by it because people won't be reluctant to refer patients for the procedure.

Wagner: What guidance can be provided on the best ways to establish and maintain lead and sheath control during the extraction procedure?

Clancy: I always tell the fellows that there's a lot of feel to the extraction. When you're pulling on it there's a lot of tactile feedback of how much tension there is. The main principle is that it's critical to keep a rail. So the laser only tracks over where we ask it to and so the better the rail, which is actually the lead we're trying to take out, keep tension on the lead and keep that lead straight because we don't want it bending or moving because the cutting tools can get off plane and when they get off plane, bad things happen. I tell the fellows they want to maintain that rail. Always keep that lead as tight and straight as possible and then that device will track over that lead and go in the appropriate direction.

\section{Wagner: How will new technology be integrated into the clinical practice?}

Clancy: I think just like AF ablation there are many different ways to approach any procedure. I think certain things will be consistent and then others will vary depending on individual practice. I think one thing that is absolutely important is that when using a rescue device, put a wire up before the start of the procedure. So the physician can have the opportunity to use the balloon if a tear occurs and if needed have the balloon over the rail if it's not up already. That way if a complication occurs, the physician doesn't run into a situation of not being able to get the wire up and so the patient is not going to be able to benefit. Then from that point once the wire is up and depending on the level of comfort for the physician, they can decide if they want the balloon available right there in case it's needed because it's a low incidence of happening or if they want to put it on the wire and have it right there. They might decide to put it inside if a patient's at higher risk. A physician might feel uneasy about the history or put it in the inferior vena cava and park it there so if needed, it's literally right there for use. I think what's going to happen going forward is what we call the workflow. How to use the balloon and the way it's set up is going to be individual operator dependent but also case dependent. We're going to reach stratified cases where this is a one year old lead and we may not really need to put a balloon in the SVC here, but this is a twenty year old lead, and even in a small young person that's high risk so we will put the balloon in. These new rescue devices give options so instead of not doing the case a physician will feel more comfortable doing the appropriate case.

\section{Wagner: What is the current state of lead extraction?}

Clancy: It's had some rapid growth mainly out of need. There's data that has come out that device infections are life threatening and the only way to really treat them appropriately in an effective way is to remove the entire system. That data has been clear and with that thought process and even the societies support, it's moving in that direction so that's increased the need for extraction and appropriately so. Then there have been several leads from a couple different companies that have had recall problems. These were leads that were not operating according to what the specifications were and putting patients at risk. So instead of adding more leads, we take those leads out and put new leads in so with that, the need has expanded the use of extraction and the need for extraction. I think it's reflected in the last five years where lead extraction even back in 2008, was a small part and now it's become a major part like AF and VT ablations and I think it speaks to the ever expanding need for appropriate patient care. 
Wagner: What major developments do you see on the horizon that will impact the field and patient care?

Clancy: I believe the Bridge ${ }^{\mathrm{TM}}$ Occlusion Balloon (Spectranetics, Colorado Springs, CO) is a remarkable development. It's unfortunate that we'll have to wait until something bad happens to use it. The balloon will perform well in these cases and we will finally have some control. From there, we have control and the ultimate goal in the field is to give the physician more tools. Maybe more diagnostic tools or the need of surgeons that do extractions can be diminished from the procedure period. We may see that even though there is an SVC tear ultimately we could do an endovascular repair. If we could safely do that and never have to crack open the chest, more patients would be appropriately referred for lead extraction.

We all have the responsibility to put in the work to make the procedure safer; to allow patients to be served better. We need to manage these patients and not let these patients suffer like they do. We put devices in these people and we have the responsibility to take care of them after that and lead management is a huge part of that. The balloon is a huge part of expanding care of these patients.

Wagner: What have physicians historically done and what has been the need to have a rescue device specifically for SVC importance?

Clancy: I've been putting up a wire for a couple years now and physicians have had that capability to use but actually really nothing has been specifically developed for SVC use. It's good to have the wire up but it's always been makeshift. There are plenty of things we do in extraction that's a little makeshift especially when we're snaring and doing femoral tools because we don't have a great set of tools available. In the past, we've tried to make do with other balloons available and they're not appropriate. The non-compliant balloons out there are not stable. Those balloons are really meant to expand and dilate everything and that's the last thing we want in an SVC tear because it could propagate the tear.

The compliancy of the Bridge ${ }^{\mathrm{TM}}$ Occlusion Balloon (Spectranetics, Colorado Springs, CO) and the development of this balloon is really to occlude the SVC and not create any more damage and to have successful use associated with minimizing blood loss. While some of the balloons are compliant, they're also small and that makes it difficult to locate the tear. The Bridge ${ }^{\mathrm{TM}}$ Occlusion Balloon (Spectranetics, Colorado Springs, CO) is different because it covers the entire SVC so it's stable and not going to cause any more injury.

It's important to emphasize that this is not a treatment. This is a compliant stabilizing device that I believe will save lives by getting a patient to the appropriate treatment which is immediate surgical intervention. This is not a replacement for all the protocols and safety that physicians already have in place. Physicians have to have an excellent system with surgeons ready to go and to anything possible to help get the patient more safely to the definitive repair. If we veer off that, we're not going to be accomplishing any goals here. Physicians need to stay true to all rescue protocols and have surgeons immediately available. I believe that the Bridge ${ }^{\mathrm{TM}}$ Occlusion Balloon (Spectranetics, Colorado Springs, CO) will do its part as an effective temporizing agent and not a definitive treatment. 